\title{
Targeting ARNT to attenuate renal fibrosis
}

\section{ce \\ modulation \\ of the \\ FKBP-YY1- \\ ARNT-ALK3 \\ signalling \\ axis may be \\ a promising \\ target in \\ chronic failure \\ of multiple \\ organ systems}

5

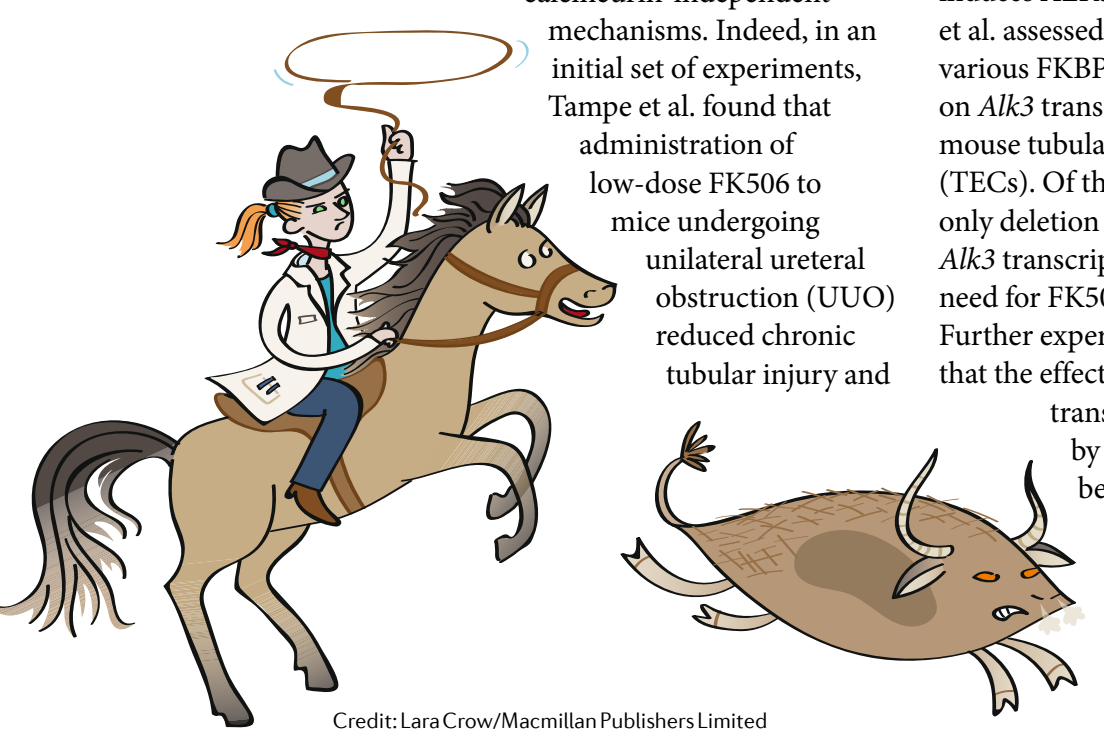

Fibrosis is an important driver of end-stage organ failure and death in a variety of chronic diseases, including chronic kidney disease. However, approaches to attenuate the progression of organ fibrosis are limited. Several studies have demonstrated efficacy of the calcineurin inhibitor (CNI) FK506 (also known as tacrolimus) in protecting against acute experimental organ injury. Now, researchers show that low-dose FK506 exerts antifibrotic, renoprotective effects in a model of renal fibrosis via aryl hydrocarbon receptor nuclear translocator (ARNT)-mediated transcription of bone morphogenetic protein receptor type $1 \mathrm{~A}$ (known herein as ALK3).

FK506 is well known for its immunosuppressive properties and is commonly used to lower the risk of rejection in organ transplant recipients. Although FK506 is nephrotoxic, studies suggest that low doses might induce renoprotective effects through calcineurin-independent mechanisms. Indeed, in an initial set of experiments, Tampe et al. found that ministration of undergoing unilateral uretera bstruction (UUO) reduced chronic by direct interactions etween FKBP12 and the transcriptional repressor protein YY1; the presence of FKBP12-YY1 complexes correlated with low Alk3 transcription, whereas depletion of FKBP12 or YY1 or the addition of FK506 induced Alk3 transcription. Moreover, TEC-specific deletion of $Y y 1$ induced Alk 3 transcription and protected mice from UUO-induced tubular injury and fibrosis.

The researchers then used array-based and computational prediction approaches to identify the transcription factor ARNT as a mediator of $A l k 3$ transcription downstream of FKBP12 and YY1. Small interfering RNA-mediated depletion of ARNT prevented FK506-induced transcription of Alk3 in vitro. Conversely, disruption of the FKBP12-YY1 complex with low-dose FK506 increased expression of Arnt in vitro and in vivo, leading to activation of $A l k 3$.

To assess the therapeutic potential of targeting the FKBP-YY1-ARNT signalling axis, the researchers used in vivo morpholinos (VMOs) targeting FKBP and YY1. Administration of these VMOs induced expression of intrarenal ARNT and protected mice from UUO-induced renal fibrosis. Similarly, a small-molecule inhibitor of FKBP12 induced intrarenal Arnt and Alk3 transcription and protected mice from tubular injury and fibrosis. Moreover, this inhibitor also attenuated organ injury in models of cardiac and liver fibrosis, prompting the researchers to speculate that modulation of the FKBP-YY1ARNT-ALK3 signalling axis may be a promising target in chronic failure of multiple organ systems.

Susan J. Allison

ORIGINAL ARTICLE Tampe, B. et al.

Pharmacological induction of hypoxia-inducible transcription factor ARNT attenuates chronic kidney failure.J Clin Invest. https://doi.org/10.1172/

Cl89632 (2018) 